\title{
Manipulating exchange bias using all-optical helicity-dependent switching
}

\author{
P. Vallobra, ${ }^{1}$ T. Fache, ${ }^{1}$ Y. Xu, ${ }_{1}^{1}$ L. Zhang, ${ }^{1}$ G. Malinowski, ${ }^{1}$ M. Hehn,,${ }^{1}$ J.-C. Rojas-Sánchez,${ }^{1}$ E. E. Fullerton, ${ }^{2}$ and S. Mangin ${ }^{1, *}$ \\ ${ }^{1}$ Institut Jean Lamour (UMR 7198), Université de Lorraine, Vandœuvre-lès-Nancy, France \\ ${ }^{2}$ Center for Memory and Recording Research, University of California San Diego, La Jolla, California 92093-0401, USA
}

(Received 18 December 2016; published 2 October 2017)

\begin{abstract}
Deterministic all-optical control of magnetization without an applied magnetic field has been reported for various materials such as ferrimagnetic and ferromagnetic thin films, as well as granular recording media. Here we demonstrate optical control of the magnetic configuration of an antiferromagnetic layer through the exchange bias interaction using the helicity of a femtosecond pulsed laser on $\mathrm{IrMn} /[\mathrm{Co} / \mathrm{Pt}]_{\mathrm{xN}}$ antiferromagnetic/ferromagnetic heterostructures. We show that the magnitude and the sign of the exchange bias field can be deterministically controlled without any applied magnetic field, only by changing the helicity of the light, the laser fluence, or the number of light pulses. We also present the combined effects of laser pulses with an applied magnetic field. This study lays the foundation for the development of new applications based on spintronic devices where the exchange bias phenomenon is routinely used to pin the magnetization orientation of a magnetic layer in one direction.
\end{abstract}

DOI: 10.1103/PhysRevB.96.144403

\section{INTRODUCTION}

Since Néel's prediction of an antiferromagnetic (AFM) spin ordering in 1936 [1] and the demonstration of AFM order in MnO by Bizette in 1938 [2], antiferromagnetism has attracted increasing attention because of fascinating physics and its major role in important and emerging applications for magnetic data storage, memories, sensors, and logic devices $[3,4]$. Most of the current functionality of AFM materials arises from the exchange bias phenomenon first observed by Meiklejohn and Bean [5] on fine particles of cobalt with a cobalt-oxide shell. The exchange bias phenomenon in AFM/ferromagnetic (FM) heterostructures manifests by a field shift in the magnetization curve of the FM layer, characterized by the exchange bias field $\left(H_{E B}\right)$. This property has been extensively used in spintronic devices such as magnetoresistive heads [6] and magnetic random access memories [7].

In current devices AFM materials are used to control and stabilize the magnetization direction of FM layers. However, AFM materials are increasingly being considered for new applications in spintronics [8-11]. This interest arises in part because AFM materials are insensitive to magnetic fields, have high intrinsic resonant frequencies in the terahertz regime, and because of the new possibility of probing and manipulating AFM layers using spin currents. For instance, it has been shown that an AFM domain wall can be moved by spin transfer torque (STT) $[3,12,13]$ and AFM order can be switched [3,14-17].

The conventional approach to establish exchange bias field consists in heating the AFM/FM bilayer structure above a certain temperature named the blocking temperature $\left(T_{B}\right)$, typically slightly smaller than the Néel temperature $\left(T_{N}\right)$. The bilayer is then cooled from $T_{B}$ under a magnetic field sufficiently high to saturate the FM layer. The orientation of the FM layer magnetization sets the orientation of the interfacial AFM spins because of the interface exchange coupling between the two layers. Other ways of controlling and manipulating exchange bias have been very recently

*Corresponding author: Stephane.mangin@univ-lorraine.fr investigated relying on ionic motion at interfaces [18] or modifying the crystal structure [14].

The fundamental mechanism explaining exchange bias has been discussed extensively [19-22] but remains a topic of high interest $[23,18]$. Ultrafast optical excitations of an AFM/FM bilayer have been used to probe the interfacial interaction between the AFM and the FM layers, and interesting fast magnetization dynamics have already been observed [24-26]. A number of reports have enlightened the fact that photoexcitation of the AFM/FM interface induces large modulations in the exchange bias field on ultrashort time scales, leading to coherent magnetization precession in the FM layers. Detailed time-resolved studies of dynamics determined that the characteristic time scale of laser-induced exchange bias quenching in a polycrystalline $\mathrm{Co} / \mathrm{IrMn}$ bilayer is $0.7 \pm 0.5 \mathrm{ps}$ [26]. In the present paper, we extended this study to femtosecond pulses and we establish that by exciting an exchange-biased system using circularly polarized femtosecond laser pulses we can deterministically control the sign of the exchange bias as well as its amplitude without any applied magnetic field.

For this study, we have grown $\mathrm{IrMn} /[\mathrm{Co} / \mathrm{Pt}]_{\mathrm{xN}}$ exchangebiased multilayers, namely, glass $/ \operatorname{Ta}(3 \mathrm{~nm}) / \operatorname{Pt}(5 \mathrm{~nm})$ $/ \operatorname{IrMn}(7 \mathrm{~nm}) /[\mathrm{Co}(0.6 \mathrm{~nm}) / \operatorname{Pt}(2 \mathrm{~nm})] \times 1 / \operatorname{Pt}(5 \mathrm{~nm})$ (sketch in Fig. 1) has been grown by dc magnetron sputtering from elemental sources onto room-temperature glass substrates. The choice was driven by the necessity to design samples combining a large perpendicular exchange bias field $[27,28]$ with the possibility to exhibit all-optical helicity-dependent switching (AOHDS) [29-33]. In [Co/Pt $] \mathrm{x}_{\mathrm{N}}$ multilayers with perpendicular magnetic anisotropy and a low number of repeats $N$, it has been demonstrated that the magnetization can be switched by either sweeping a femtosecond laser beam [32] or using a static beam [34]. Indeed it was previously shown that for $[\mathrm{Co}(0.6 \mathrm{~nm}) / \operatorname{Pt}(2 \mathrm{~nm})] \mathrm{x}_{\mathrm{N}}$ with $N \leqslant 2$ the criteria related to stable domain size needed to observe AOHDS is fulfilled [35]. Note that for AOHDS with either a sweeping beam or a static beam, multiple laser pulses are needed to deterministically reverse the magnetization [36]. For this study, we used the experimental setup described in Refs. [32-34].

As shown in Fig. 2, in exchange-biased systems, the hysteresis loop (magnetization vs applied magnetic field $H$ ) 


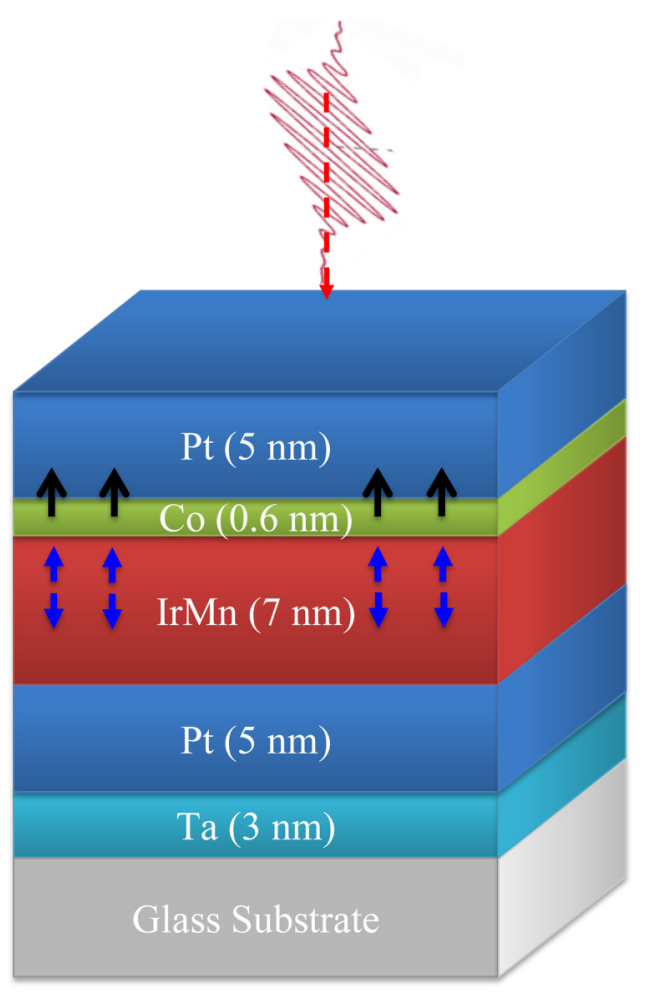

FIG. 1. Schematic of the interplay between femtosecond pulses and exchange bias for an $\operatorname{IrMn}(7 \mathrm{~nm}) /[\operatorname{Co}(0.6 \mathrm{~nm}) / \operatorname{Pt}(2 \mathrm{~nm})]$ multilayer showing perpendicular exchange bias.

of the FM layer is characterized by its width, the coercive field $\left(H_{C}\right)$, and its horizontal shift along the field axis, the exchange bias field $\left(H_{E B}\right)$. In our samples, the IrMn thickness has been optimized to observe a maximum exchange bias effect, and two samples with $N=2$ (Fig. 3) and $N=1$ (Fig. 2) have been chosen to carry out the study of the effect of polarized femtosecond laser pulses on the magnetic configuration of the bilayer. In the as-grown state, a small exchange bias field is observed with $N=1$ [Fig. 2(b), $H_{C}=34.9 \mathrm{mT}$, and $\left.H_{E B}=-11.1 \mathrm{mT}\right]$. Such a small exchange effect without any annealing process has already been reported in $[37,38]$.

\section{AOHDS REVERSAL AND CONTROL OF EXCHANGE BIAS}

First we performed experiments similar to that used to study AOHDS in thin ferromagnetic films. The laser illuminated the Pt capping layer to determine the ability to optically control the orientation of the FM layer magnetization when exchange biased with the AFM layer.

To perform optical excitation, we use a Ti:sapphire femtosecond laser with a $5-\mathrm{kHz}$ repetition rate, a wavelength of $800 \mathrm{~nm}(1.55 \mathrm{eV})$, and a pulse duration of $35 \mathrm{fs}$. The Gaussian beam spot is focused with a FWHM of approximately $50 \mu \mathrm{m}$ and swept with a velocity of about $5 \mu \mathrm{m} / \mathrm{s}$. The response of the magnetic film was studied using a static Faraday microscope to image the magnetic domains while the laser is illuminating the sample. A zero-order quarter-wave plate, which transforms linearly polarized light (L) into left-handed $\left(\sigma^{+}\right)$or right-handed $\left(\sigma^{-}\right)$circularly polarized light, controls

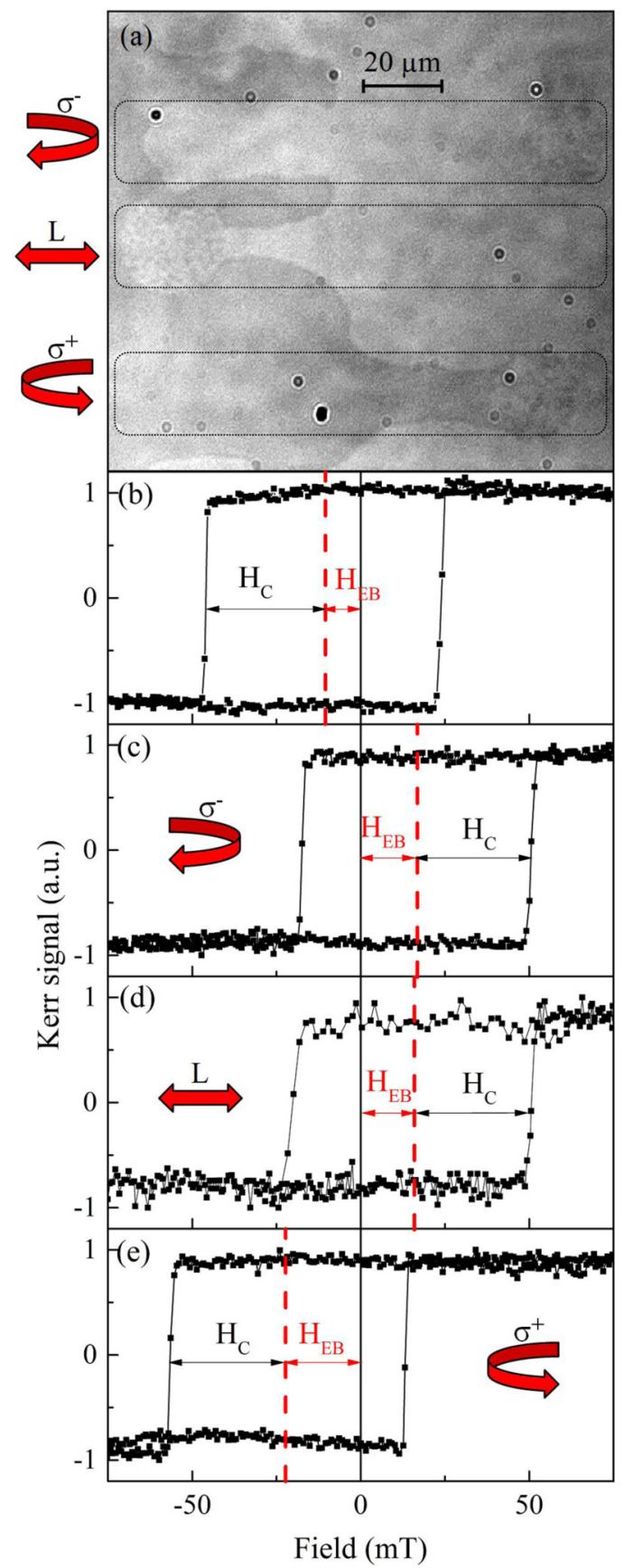

FIG. 2. Results obtained on an exchange-biased sample: glass/Ta(3 nm)/Pt(5 nm)/IrMn(7 nm)/[Co(0.6 nm)/Pt(2 nm)]× $1 / \operatorname{Pt}(5 \mathrm{~nm})$. (a) Faraday imaging after that, a right-circularly $\left(\sigma^{-}\right)$, a linear $L$, and a left-circularly $\left(\sigma^{+}\right)$polarized laser beam have been swept over the sample with a sweeping speed of approximately 10 $\mu \mathrm{m} / \mathrm{s}$. Kerr signal hysteresis loop obtained on (b) an unexposed sample area (as-grown sample), (c) on an area where a right-circularly polarized $\left(\sigma^{-}\right)$laser beam was swept, (d) area where a linearly polarized $(L)$ laser beam was swept, and (e) area where a leftcircularly polarized $\left(\sigma^{+}\right)$laser beam was swept. For all expositions to the laser the fluence was $8.9 \mathrm{~mJ} / \mathrm{cm}^{2}$.

the helicity of the beam. The present measurements are performed at room temperature, and the laser beam was swept at a constant rate of 3-20 $\mu \mathrm{ms}^{-1}$ with a typical laser spot size of $50 \mu \mathrm{m}$. Magnetic images were obtained in transmission using 


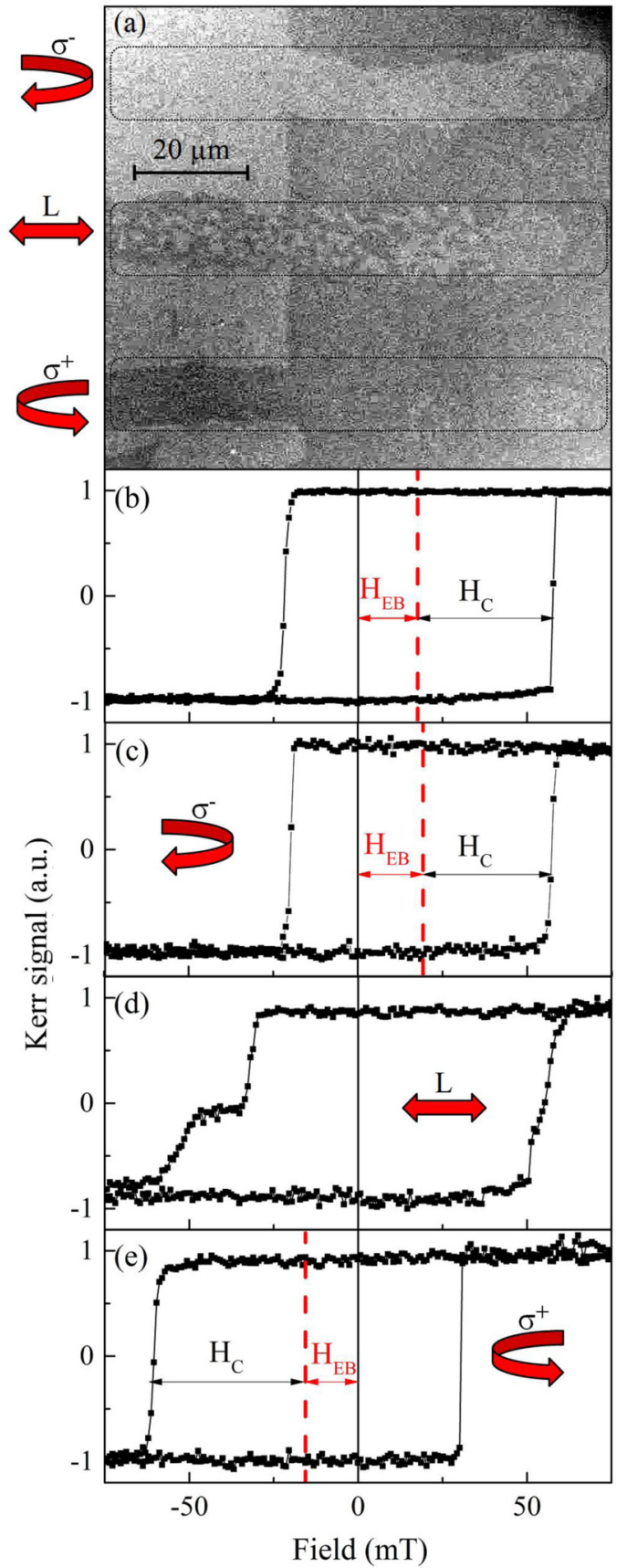

FIG. 3. Results obtained on an exchange-biased sample: glass $/ \mathrm{Ta}(3 \mathrm{~nm}) / \operatorname{Pt}(5 \mathrm{~nm}) / \operatorname{IrMn}(7 \mathrm{~nm}) /[\mathrm{Co}(0.6 \mathrm{~nm}) / \operatorname{Pt}(2 \mathrm{~nm})] \times$ $2 / \operatorname{Pt}(5 \mathrm{~nm})$. (a) Faraday imaging after that, a right-circularly $\left(\sigma^{-}\right)$, a linear $L$ and a left-circularly $\left(\sigma^{+}\right)$polarized laser beam have been swept over the sample with a sweeping speed of approximately 10 $\mu \mathrm{m} / \mathrm{s}$. Kerr signal hysteresis loop obtained on (b) an unexposed sample area (as-grown sample), (c) on an area where a right-circularly polarized, $\left(\sigma^{-}\right)$laser beam was swept, (d) area where a linearly polarized $(L)$ laser beam was swept, and (e) area where a leftcircularly polarized, $\left(\sigma^{+}\right)$laser beam was swept. For all expositions to the laser the fluence was $8.9 \mathrm{~mJ} / \mathrm{cm}^{2}$.

a magneto-optic Faraday microscope. Magnetic hysteresis measurements were performed using a magneto-optic Kerr effect setup. The background signal was subtracted from the initial hysteresis loops.
As shown in Fig. 2(a), the final state of the sample is defined by the helicity of the circularly polarized light when the laser beam is swept on the sample similarly to what was observed for $\mathrm{Co} / \mathrm{Pt}$ multilayers [32]. These results demonstrate that AOHDS of the $[\mathrm{Co} / \mathrm{Pt}]$ multilayer is observed even with the additional exchange coupling with the IrMn (AFM) layer.

In addition to the reversal of the FM magnetization orientation, the sign of the exchange bias is determined by the AOHDS process. The hysteresis loops reported in Figs. 2(c)-2(e) were measured on areas where the laser beam was swept with $\sigma^{-}, L$, and $\sigma^{+}$polarizations, respectively. This shows without any ambiguity that $\sigma^{+}$polarized light induces a negative exchange bias whereas $\sigma^{-}$polarized light induces a positive exchange bias. After sweeping the laser, the bias direction is consistent with the final state of the $[\mathrm{Co} / \mathrm{Pt}]_{\mathrm{xN}}$ magnetization, independently of the initial bias direction. The process is reversible and the sign of the exchange bias for the same area of the sample can be reversed with subsequent sweeping of the beam with opposite helicity. In all cases the exchange bias effect is set in agreement with the FM domain orientation induced by the AOHDS process.

\section{STATIC BEAM EXPERIMENTS}

Static beam experiments with the same fluence as the one used in Fig. 2 have been performed by varying the number of pulses from 1 to 50000. Starting from the as-grown sample, the $\sigma^{-}$helicity was used and local hysteresis loops were measured at different positions inside and outside the disk optically drawn by the laser spot (Fig. 4). Since the laser intensity is larger in the center of the spot and decreases as one moves away from its center, it allows us to study the

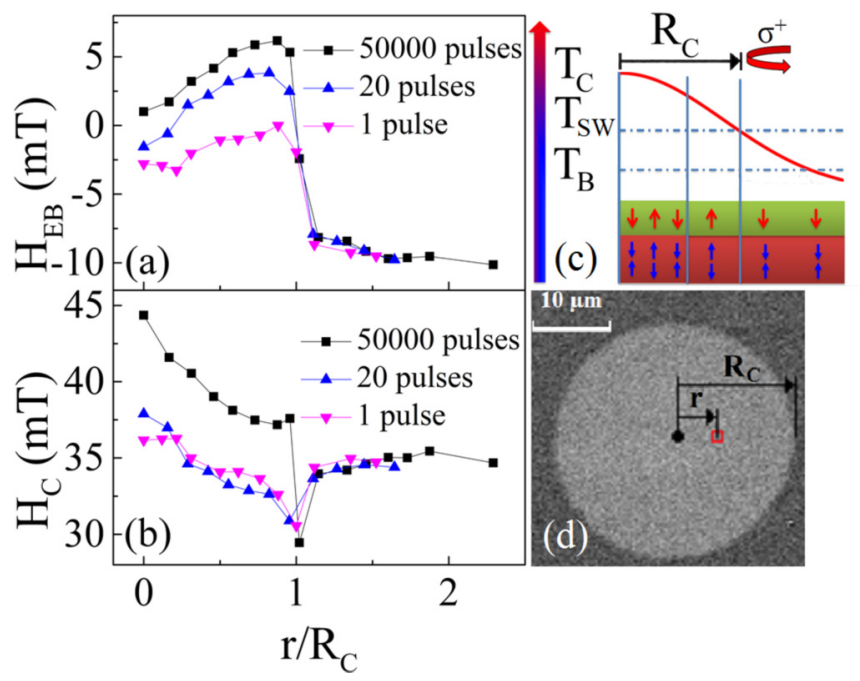

FIG. 4. Results obtained on glass/Ta(3 nm)/Pt(5 nm)/IrMn $(7 \mathrm{~nm}) /[\mathrm{Co}(0.6 \mathrm{~nm}) / \mathrm{Pt}(2 \mathrm{~nm})] \times 1 / \operatorname{Pt}(5 \mathrm{~nm})$. (a) Exchange bias field $\left(H_{E B}\right)$ and $(\mathrm{b})$ coercive field $\left(H_{C}\right)$ as a function of the position $(r)$ from the center of a laser spot after a single pulse (full triangles pointing up), 20 pulses (full triangles pointing down), and after 50000 pulses (full squares). (c) Sketch of the laser intensity profile and the corresponding magnetic configuration. (d) Magneto-optical Kerr effect microscope image of the sample after a single laser pulse. The fluence of the laser was $8.9 \mathrm{~mJ} / \mathrm{cm}^{2}$. 
influence of the laser intensity and the number of pulses on the induced exchange bias. Figure 4 reports the evolution of the exchange bias field (a) and the coercive field (b) as a function of the distance from the circle center after 1, 20, and 50000 pulses.

First, the results show that for a radius $(r)$ larger than a critical value $\left(R_{C}\right)$ the effect of light on the exchange bias and the coercivity is negligible. In other words, there is a critical intensity $\left(I_{C}\right)$ below which the exchange bias state is unaffected by the optical excitation. The largest exchange bias field change is observed for an intensity just above $I_{C}$, where $r$ is slightly smaller than $R_{C}$. For a single pulse the exchange bias field is significantly reduced, while for a large number of pulses (20 and 50000) the sign of the exchange bias field is deterministically changed from negative to positive for the $\sigma^{-}$helicity. These measurements imply that multiple pulses are needed to control the sign of the exchange bias field. This is consistent with measurements showing that AOHDS in $\mathrm{Co} / \mathrm{Pt}$ multilayers requires multiple pulses [36]. As sketched in Fig. 4(c), at the center of the beam the intensity is high enough to lead to $\mathrm{Co} / \mathrm{Pt}$ demagnetization, in agreement with previous measurements [32]. Consequently, when the laser is switched off, the orientation of the interfacial magnetic moments in the IrMn follows the local $\mathrm{Co} / \mathrm{Pt}$, leading to zero exchange bias. For $r$ close to $R_{C}$ a "switching ring" is reached where the laser can switch deterministically the ferromagnetic layer and therefore, through the exchange coupling, rearranges the IrMn magnetic moments at the interface, thus leading to a change in the exchange bias field. The maximum of the effect is observed for $(r=R c)$. For $r>R c$ the light has no effect on the magnetization of the ferromagnet.

\section{SWEEPING BEAM EXPERIMENTS}

While magnetic switching is observed in both static and sweeping beam experiments, the exchange bias field values measured after the static multiple pulses process are smaller than those obtained by sweeping the laser beam over the sample. This result could be understood considering the different characteristic temperatures of the AFM/FM system and the time needed to cross those temperatures.

From the highest temperatures towards room temperature, the AFM/FM system has to cross first the Curie temperature $\left(T_{C}\right)$ of the $\mathrm{Co} / \mathrm{Pt}$ multilayer, found to be above $640 \mathrm{~K}$ for the considered $\mathrm{Co}$ and $\mathrm{Pt}$ thicknesses [39]. From previous measurements [30] it appears that another temperature $T_{S W}$ slightly smaller than $T_{C}$ could be defined as the temperature at which the light has the largest effect on the magnetization switching of the FM layer. Finally, the IrMn AFM layer blocking temperature, the temperature at which the exchange bias is equal to zero, is $T_{B}=420 \mathrm{~K}$ [40].

Starting with a FM magnetization pointing down and a positive exchange bias, the laser is swept towards the right with a polarization that reverses the magnetization of the FM layer, as sketched in Fig. 5. In region (A) the laser fluence is sufficiently small that the temperature is lower than $T_{B}$. The laser has no impact on the magnetic configuration, a situation that is similar to $r>R_{C}$ in Fig. 4. In region (B) the temperature exceeds $T_{B}$. The AFM layer starts to be disoriented, whereas the FM layer remains unaffected. In region (C) the laser fluence

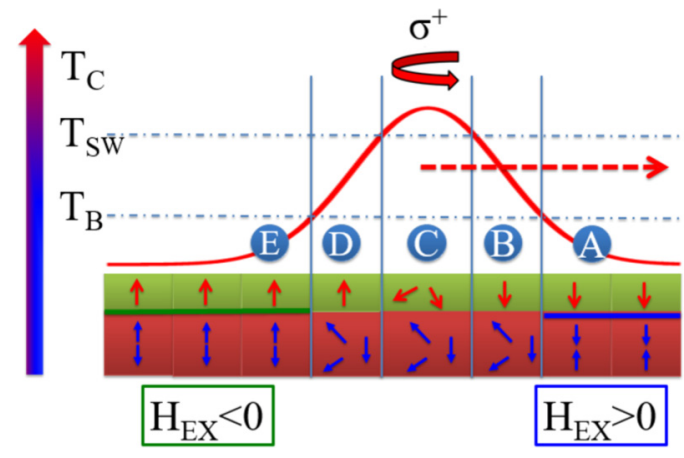

FIG. 5. Sketch of the effect of a sweeping beam on the AFM/FM magnetic configuration. In (A) the laser power is too low to influence either the AFM or FM magnetic configuration. Initially, the FM magnetization is pointing down and the exchange bias is positive. For (B) the power is large enough to demagnetize the AFM. (C) The left-circular polarization $\left(\sigma^{+}\right)$and the laser fluence allows the FM switching from down to up while the temperature is above the AFM blocking temperature. (D) The AFM layer is cooled down through its blocking temperature with a FM layer magnetization pointing up, which leads to a negative exchange bias.

and helicity is sufficient to reach $T_{S W}$ and to reverse the $\mathrm{Co} / \mathrm{Pt}$ magnetization. In regions (D) to (E) the bilayer cools down through $T_{B}$ with a reversed magnetized FM layer which reorients the AFM layer and so the exchange bias. Thus the orientation of the AFM is not set directly by the helicity of the light but by the orientation of the FM while crossing the blocking temperature.

As already highlighted, the mechanism involved in the magnetization dynamic induced by femtosecond laser pulses can be described with three interacting reservoirs (electrons, spins, and lattice) [41]. Consequently, three temperatures can be defined: the electron $T_{E}$, the spin $T_{S}$, and the lattice $T_{L}$ temperature. When comparing the estimation of those temperatures given in Refs. [42] and [43] with the one governing the setting of the exchange bias $\left(T_{B}\right)$, unexpectedly, only electrons and spins could reach this temperature. Therefore it would imply that the exchange bias can be set by an increase of the electron and spin temperatures during a few picoseconds. Furthermore, the setting of the exchange bias using a conventional annealing process is modeled by thermal activation of the AFM grains [44]; the kinetics are supposed to be much slower. In conclusion, either the lattice reaches the blocking temperature or a new description is required to explain the ultrafast exchange bias setting.

The differences between the static and sweeping beam responses could be due to the fact that $T_{C}, T_{S W}$, and $T_{B}$ are close. Indeed, while sweeping the FM layer has time to be thermalized and saturated when the temperature of IrMn crosses $T_{B}$, leading to a high exchange bias field. On the contrary, in the static experiment when the electronic temperature of IrMn crosses $T_{B}$ the magnetization of the FM layer is still fluctuating, leading to a reduced exchange bias.

Finally, to confirm the role of the orientation of the FM layer and the cooling process, we studied the combined effects of the laser and an applied magnetic field. Figure 6 compares 


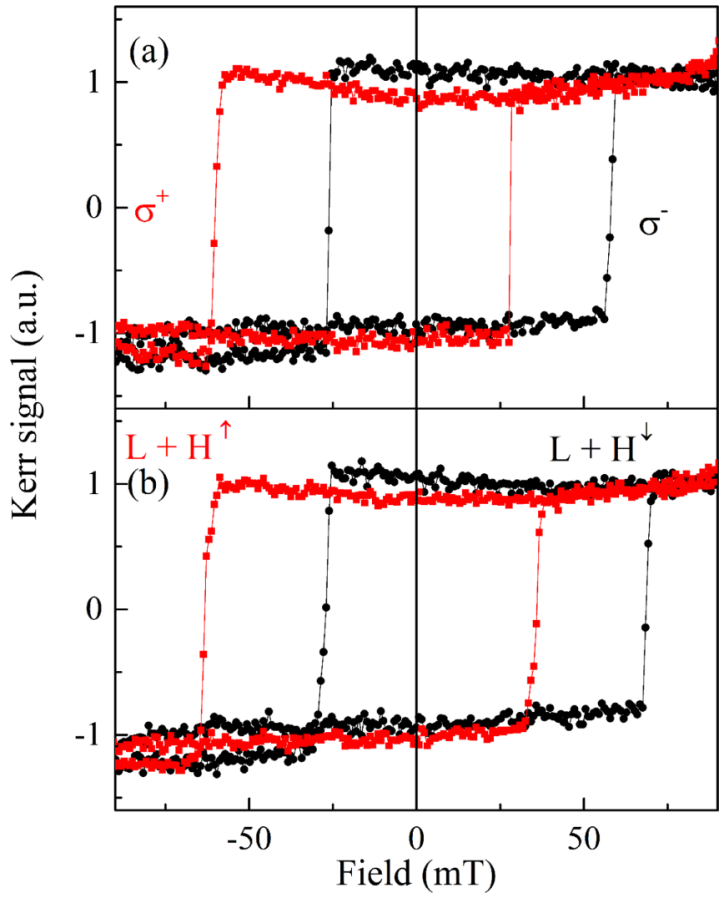

FIG. 6. Hysteresis loop of glass/Ta(3 nm)/Pt(5 nm)/IrMn $(7 \mathrm{~nm}) /[\mathrm{Co}(0.6 \mathrm{~nm}) / \mathrm{Pt}(2 \mathrm{~nm})] \times 1 / \mathrm{Pt}(5 \mathrm{~nm})$. Comparison between (a) the effect of a right-circularly $\left(\sigma^{-}\right)$or a left-circularly $\left(\sigma^{+}\right)$polarized laser beam, with (b) the combined effects of a linearly polarized light and an applied magnetic field, up or down. The fluence of the laser was $8.9 \mathrm{~mJ} / \mathrm{cm}^{2}$.

the effect of sweeping a circularly polarized laser beam [Fig. 6(a)] with sweeping a linearly polarized laser beam while a magnetic field sufficiently large to saturate the magnetization is applied [Fig. 6(b)]. This demonstrates that the effect of $\sigma^{+}$[resp. $\sigma^{-}$] polarization on the exchange bias field and coercive field is similar to the effect of a linear polarization and a positive $(\mathrm{H} \uparrow)$ [resp. negative $(\mathrm{H} \downarrow)$ ] applied magnetic fields.

In the second experiment, the laser was swept with fluence such that it does not affect the orientation of the $\mathrm{Co} / \mathrm{Pt}$ layer magnetization. The magnetization was set either up or down by an applied magnetic field and then the laser was scanned with a reduced laser intensity. As shown in Fig. 7, the resulting exchange bias is then set by the orientation of the $\mathrm{Co} / \mathrm{Pt}$ magnetization before the light exposure and no significant influence of the helicity of the light is observed. In that case the antiferromagnetic configuration is modified by the combined heating effects of the laser and the initial orientation of the $\mathrm{Co} / \mathrm{Pt}$ layer.

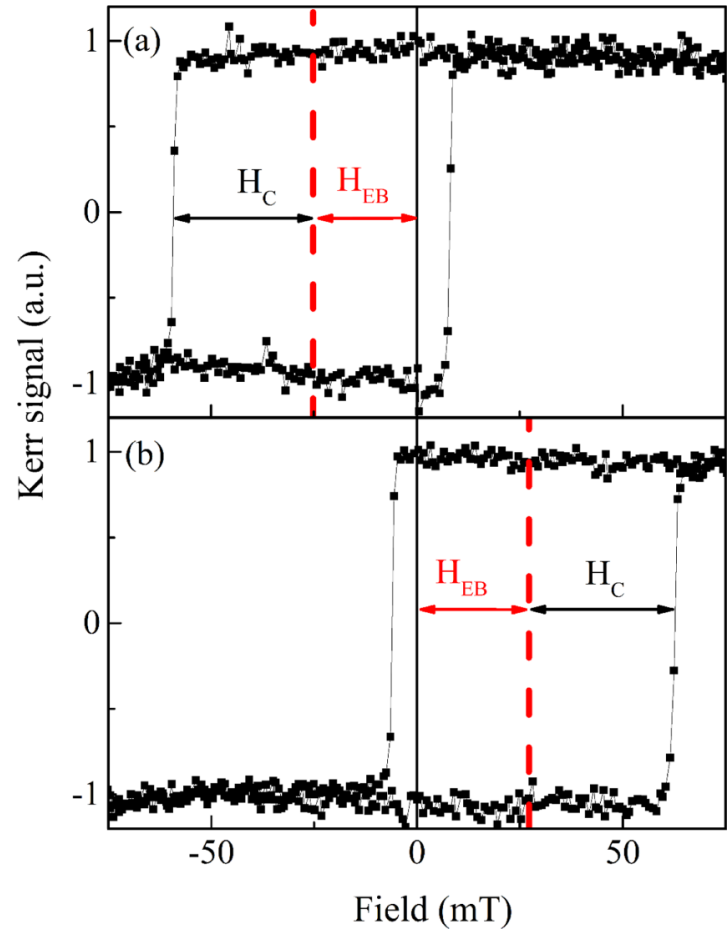

FIG. 7. Hysteresis loop of glass/Ta(3 nm)/Pt(5 nm)/IrMn $(7 \mathrm{~nm}) /[\mathrm{Co}(0.6 \mathrm{~nm}) / \mathrm{Pt}(2 \mathrm{~nm})] \times 1 / \mathrm{Pt}(5 \mathrm{~nm})$ obtained in an area where a linearly polarized laser with a fluence just under the switching threshold $\left(5.7 \mathrm{~mJ} / \mathrm{cm}^{2}\right)$ has been swept beginning from (a) saturation down and (b) saturation up.

\section{CONCLUSION}

In conclusion, we have demonstrated that exchange bias can be manipulated with femtosecond laser pulses without any applied field. We showed that the AFM magnetic configuration can be modified deterministically but indirectly with light only. The femtosecond polarized laser first modifies the FM orientation, which sets the exchange bias while cooling. This effect takes place while the electronic temperature is above the blocking temperature during a few picoseconds. The next perspective consists in determining if the AFM layer can be directly manipulated without the help of a FM layer but with the helicity of the light like the AOHDS in some ferromagnets and ferrimagnets.

In terms of applications, our work may have a strong impact on magnetic memories, logic, and recording technologies. The possibility to manipulate the exchange bias coupling locally, as it was already suggested for the thermally activated switching magnetoresistive random access memory (MRAM) concept [45], would offer scalability, thermal stability, energy efficiency, and low response to residual fields.
[1] L. Néel, Influence des fluctuations du champ moléculaire sur les propriétés magnétiques des corps, Ann. Phys. 10, 5 (1932).

[2] H. Bizette, C. F. Squire, and B. Tsai, The $\lambda$ transition point of the magnetic susceptibility in the manganosite $\mathrm{MnO}, \mathrm{C}$. R. Acad. Sci., Paris 207, 449 (1938).
[3] T. Jungwirth, X. Marti, P. Wadley, and J. Wunderlich, Antiferromagnetic spintronics, Nat. Nano. 11, 231 (2016).

[4] P. Wadley, B. Howells, J. Zelezny, C. Andrews, V. Hills, R. P. Campion, V. Novak, F. Freimuth, Y. Mokrousov, A. W. Rushforth, K. W. Edmonds, B. L. Gallagher, and T. Jungwirth, 
Electrical switching of an antiferromagnet, Science 351, 587 (2016).

[5] W. H. Meiklejohn and C. P. Bean, New magnetic anisotropy, Phys. Rev. 105, 904 (1957).

[6] E. E. Fullerton and J. R. Childress, Spintronics, magnetoresistive heads, and the emergence of the digital world, Proc. IEEE 104, 1787 (2016).

[7] D. Apalkov, B. Dieny, and J. M. Slaughter, Magnetoresistive random access memory, Proc. IEEE 104, 1796 (2016).

[8] A. S. Nunez, R. A. Duine, P. Haney, and A. H. MacDonald, Theory of spin torques and giant magnetoresistance in antiferromagnetic metals, Phys. Rev. B 73, 214426 (2006).

[9] P. M. Haney and A. H. MacDonald, Current-Induced Torques Due to Compensated Antiferromagnets, Phys. Rev. Lett. 100, 196801 (2008).

[10] H. V. Gomonay and V. M. Loktev, Spin transfer and currentinduced switching in antiferromagnets, Phys. Rev. B 81, 144427 (2010).

[11] E. Y. Vedmedenko, K. von Bergmann, A. Kubetzka, P. Ferriani, S. Heinze, and R. Wiesendanger, Atomic spin structure of antiferromagnetic domain walls, Nat. Mater. 5, 477 (2006).

[12] R. Cheng and Q. Niu, Dynamics of antiferromagnets driven by spin current, Phys. Rev. B 89, 081105(R) (2014).

[13] E. G. Tveten, A. Qaiumzadeh, and A. Brataas, Antiferromagnetic Domain Wall Motion Induced by Spin Waves, Phys. Rev. Lett. 112, 147204 (2014).

[14] W. Yuan, T. Su, Q. Song, W. Xing, Y. Chen, T. Wang, Z. Zhang, X. Ma, P. Gao, J. Shi, and W. Han, Crystal structure manipulation of the exchange bias in an antiferromagnetic film, Sci. Rep. 6, 28397 (2016).

[15] X.-L. Tang, H.-W. Zhang, H. Su, Z.-Y. Zhong, and Y.-L. Jing, Changing and reversing the exchange bias in a current-in-plane spin valve by means of an electric current, Appl. Phys. Lett. 91, 122504 (2007).

[16] H. Sakakibara, H. Ando, Y. Kuroki, S. Kawai, K. Ueda, and H. Asano, Magnetic properties and anisotropic magnetoresistance of antiperovskite nitride $\mathrm{Mn}_{3} \mathrm{GaN} / \mathrm{Co}_{3} \mathrm{FeN}$ exchange-coupled bilayers, J. Appl. Phys. 117, 17D725 (2015).

[17] X. He, Y. Wang, N. Wu, A. N. Caruso, E. Vescovo, K. D. Belaschenko, P. A. Dowben, and C. Binek, Robust isothermal electric control of exchange bias at room temperature, Nat. Mater. 9, 579 (2010).

[18] D. A. Gilbert, J. Olamit, R. K. Dumas, B. J. Kirby, A. J. Grutter, B. B. Maranville, E. Arenholz, J. A. Borchers, and K. Liu, Controllable positive exchange bias via redox-driven oxygen migration, Nat. Commun. 7, 11050 (2016).

[19] J. Nogués and I. K. Schuller, Exchange bias, J. Magn. Magn. Mater. 192, 203 (1999).

[20] A. E. Berkowitz and K. Takano, Exchange anisotropy-a review, J. Magn. Magn. Mater. 200, 552 (1999).

[21] R. L. Stamps, Mechanisms for exchange bias, J. Phys. D 33, R247 (2000).

[22] G. Malinowski, M. Hehn, S. Robert, O. Lenoble, A. Schuhl, and P. Panissod, Magnetic origin of enhanced top exchange biasing in Py/Irmn/Py multilayers, Phys. Rev. B 68, 184404 (2003).

[23] M. Gruber, F. Ibrahim, S. Boukari, H. Isshiki, L. Joly, M. Peter, M. Studniarek, V. Da Costa, H. Jabbar, V. Davesne, U. Halisdemir, J. Chen, J. Arabski, E. Otero, F. Choueikani, K. Chen, P. Ohresser, W. Wulfhekel, F. Scheurer, W. Weber,
M. Alouani, E. Beaurepaire, and M. Bowen, Exchange bias and room-temperature magnetic order in molecular layers, Nat. Mater. 14, 981 (2015).

[24] G. Ju, A. V. Nurmikko, R. F. C. Farrow, R. F. Marks, M. J. Carey, and B. A. Gurney, Ultrafast Time Resolved Photoinduced Magnetization Rotation in a Ferromagnetic/Antiferromagnetic Exchange Coupled System, Phys. Rev. Lett. 82, 3705 (1999).

[25] A. Porat, S. Bar-Ad, and I. K. Schuller, Novel laser-induced dynamics in exchange-biased systems, Europhys. Lett. 87, 67001 (2009).

[26] F. Dalla Longa, J. T. Kohlhepp, W. J. M. de Jonge, and B. Koopmans, Resolving the genuine laser-induced ultrafast dynamics of exchange interaction in ferromagnet/antiferromagnet bilayers, Phys. Rev. B 81, 094435 (2010).

[27] S. Maat, K. Takano, S. S. P. Parkin, and E. E. Fullerton, Perpendicular Exchange Bias of Co/Pt Multilayers, Phys. Rev. Lett. 87, 087202 (2001).

[28] J. Sort, V. Baltz, F. Garcia, B. Rodmacq, and B. Dieny, Tailoring perpendicular exchange bias in [Pt/Co]-IrMn multilayers, Phys. Rev. B 71, 054411 (2005).

[29] C. D. Stanciu, F. Hansteen, A. V. Kimel, A. Kirilyuk, A. Tsukamoto, A. Itoh, and Th. Rasing, All-Optical Magnetic Recording with Circularly Polarized Light, Phys. Rev. Lett. 99, 047601 (2007).

[30] S. Alebrand, M. Gottwald, M. Hehn, D. Steil, M. Cinchetti, D. Lacour, E. E. Fullerton, M. Aeschlimann, and S. Mangin, Light-induced magnetization reversal of high-anisotropy $\mathrm{TbCo}$ alloy films, Appl. Phys. Lett. 101, 162408 (2012).

[31] A. R. Khorsand, M. Savoini, A. Kirilyuk, A. V. Kimel, A. Tsukamoto, A. Itoh, and T. Rasing, Role of Magnetic Circular Dichroism in All-Optical Magnetic Recording, Phys. Rev. Lett. 108, 127205 (2012).

[32] C. H. Lambert, S. Mangin, B. S. D. Ch. S. Varaprasad, Y. K. Takahashi, M. Hehn, M. Cinchetti, G. Malinowski, K. Hono, Y. Fainman, M. Aesclimann, and E. E. Fullerton, All-optical control of ferromagnetic thin films and nanostructures, Science 345, 1337 (2014).

[33] S. Mangin, M. Gottwald, C.-H. Lambert, D. Steil, L. Pang, M. Hehn, S. Alebrand, M. Cinchetti, G. Malinowski, Y. Fainman, M. Aeschlimann, and E. E. Fullerton, Engineered materials for all-optical helicity-dependent magnetic switching, Nat. Mater. 13, 286 (2014).

[34] M. S. El Hadri, P. Pirro, C. H. Lambert, N. Bergeard, S. PetitWatelot, M. Hehn, G. Malinowski, Y. Quessab, R. Medapalli, E. E. Fullerton, and S. Mangin, Electrical characterization of all-optical helicity-dependent switching in ferromagnetic Hall crosses, Appl. Phys. Lett. 108, 092405 (2016).

[35] M. S. El Hadri, M. Hehn, P. Pirro, C.-H. Lambert, G. Malinowski, E. E. Fullerton, and S. Mangin, Domain size criterion for the observation of all-optical helicity-dependent switching in magnetic thin films, Phys. Rev. B 94, 064419 (2016).

[36] M. S. El Hadri, P. Pirro, C. H. Lambert, S. Petit-Watelot, Y. Quessab, M. Hehn, F. Montaigne, G. Malinowski, and S. Mangin, Two types of all-optical magnetization switching mechanisms using femtosecond laser pulses, Phys. Rev. B 94, 064412 (2016).

[37] S. Van Dijken, J. Moritz, and J. M. D. Coey, Correlation between perpendicular exchange bias and magnetic anisotropy 
in $\mathrm{IrMn} /[\mathrm{Co} / \mathrm{Pt}]_{\mathrm{n}}$ and $[\mathrm{Pt} / \mathrm{Co}]_{\mathrm{n}} / \mathrm{IrMn}$ multilayers, J. Appl. Phys. 97, 063907 (2005).

[38] G. Anderson, Y. Huai, and L. Miloslawsky, CoFe/IrMn exchange biased top, bottom, and dual spin valves, J. Appl. Phys. 87, 6989 (2000).

[39] H. W. van Kesteren and W. B. Zeper, Controlling the Curie temperature of $\mathrm{Co} / \mathrm{Pt}$ multilayer magneto-optical recording media, J. Magn. Magn. Mater. 120, 271 (1993).

[40] V. Baltz, Thermally driven asymmetric responses of grains versus spin-glass related distributions of blocking temperature in exchange bias Co/IrMn bilayer, Appl. Phys. Lett. 102, 062410 (2013).

[41] E. Beaurepaire, J.-C. Merle, A. Daunois, and J.-Y. Bigot, Ultrafast Spin Dynamics in Ferromagnetic Nickel, Phys. Rev. Lett. 76, 4250 (1996).
[42] A. Mekonnen, A. R. Khorsand, M. Cormier, A. V. Kimel, A. Kirilyuk, A. Hrabec, L. Ranno, A. Tsukamoto, A. Itoh, and T. Rasing, Role of the inter-sublattice exchange coupling in short-laser-pulse-induced demagnetization dynamics of $\mathrm{GdCo}$ and GdCoFe alloys, Phys. Rev. B 87, 180406(R) (2013).

[43] M. Vomir, R. Turnbull, I. Birced, P. Parreira, D. A. MacLaren, S. Lee, P. André, and J.-Y. Bigot, Dynamical torque in $\mathrm{Co}_{x} \mathrm{Fe}_{3-x} \mathrm{O}_{4}$ nano-cube thin films characterized by femtosecond magnetooptics: A $\pi$-shift control of the magnetization precession, Nano Lett. 16, 5291 (2016).

[44] T. Hugues, K. O’Grady, H. Laidler, and R. W. Cantrell, Thermal activation in exchange biased bilayers, J. Magn. Magn. Mater. 235, 329 (2001).

[45] I. L. Prejbeanu, M. Kerekes, R. C. Sousa, H. Sibuet, O. Redon, B. Dieny, and J. P. Nozières, Thermally assisted MRAM, J. Phys.: Condens. Matter 19, 16 (2007). 